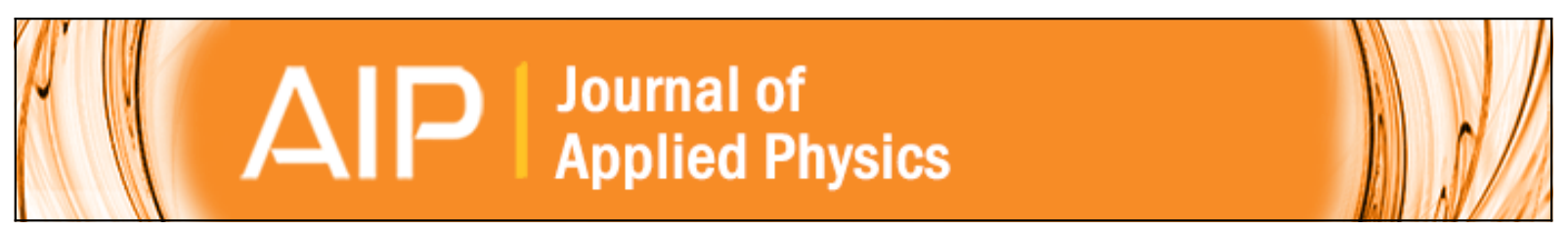

\title{
Solitary waves in a chain of repelling magnets
}

Miguel Molerón, Andrea Leonard, and Chiara Daraio

Citation: Journal of Applied Physics 115, 184901 (2014); doi: 10.1063/1.4872252

View online: http://dx.doi.org/10.1063/1.4872252

View Table of Contents: http://scitation.aip.org/content/aip/journal/jap/115/18?ver=pdfcov

Published by the AIP Publishing

\section{Articles you may be interested in}

Topographically induced internal solitary waves in a pycnocline: Primary generation and topographic control

Phys. Fluids 25, 066601 (2013); 10.1063/1.4808163

Numerical simulation of mass transport in internal solitary waves

Phys. Fluids 24, 016602 (2012); 10.1063/1.3676771

Interaction of a large amplitude interfacial solitary wave of depression with a bottom step

Phys. Fluids 22, 076602 (2010); 10.1063/1.3455984

2D Solitary Waves of Boussinesq Equation

AIP Conf. Proc. 755, 85 (2005); 10.1063/1.1900395

Nonanalytic nonlinear oscillations: Christiaan Huygens, quadratic Schrödinger equations, and solitary waves J. Acoust. Soc. Am. 104, 1289 (1998); 10.1121/1.424338

\section{A|P| $\left.\right|_{\text {Applied Physics }} ^{\text {Journal of }}$}

Journal of Applied Physics is pleased to announce André Anders as its new Editor-in-Chief 


\title{
Solitary waves in a chain of repelling magnets
}

\author{
Miguel Molerón, ${ }^{1}$ Andrea Leonard, ${ }^{1,2}$ and Chiara Daraio ${ }^{1,2, a)}$ \\ ${ }^{1}$ Department of Mechanical and Process Engineering, Swiss Federal Institute of Technology (ETH), \\ Zurich, Switzerland \\ ${ }^{2}$ Division of Engineering and Applied Science, California Institute of Technology, Pasadena, \\ California 91125, USA
}

(Received 5 March 2014; accepted 11 April 2014; published online 8 May 2014)

\begin{abstract}
We study experimentally, numerically, and theoretically the dynamics of a one dimensional array of repelling magnets. We demonstrate that such systems support solitary waves with a profile and propagation speed that depend on the amplitude. The system belongs to the kind of nonlinear lattices studied in [Friesecke and Matthies, Physica D 171, 211-220 (2002)] and exhibits a sech ${ }^{2}$ profile in the low energy regime and atomic scale localization in the high energy regime. Such systems may find potential applications in the design of novel devices for shock absorption, energy localization and focusing. Furthermore, due to the similarity of the magnetic potential with the potentials governing atomic forces, the system could be used for a better understanding of important problems in physics and chemistry. (C) 2014 AIP Publishing LLC. [http://dx.doi.org/10.1063/1.4872252]
\end{abstract}

\section{INTRODUCTION}

Since the pioneering works of Fermi, Pasta, and Ulam ${ }^{1}$ and of Zabusky and $\mathrm{Kruskal}^{2}$ the dynamics of nonlinear lattices (NLs) has received unceasing attention and has motivated extensive research (see Refs. 3 and 4 for a review).

NLs are often represented by nonlinear mass-spring systems. In spite of its relative simplicity, such systems are of great interest both from fundamental and practical points of view. NLs are used to elucidate features of atomic and molecular chains. ${ }^{5-7}$ They are also excellent testbeds in which fundamental aspects of nonlinear dynamics can be investigated, such as second-harmonic generation, ${ }^{8,9}$ nonlinear resonances, ${ }^{8}$ chaos and bifurcations, ${ }^{10}$ as well as the formation of traveling solitary waves and breathers. ${ }^{1-13}$ In the last decade, exploiting the nonlinearities of such systems has led to the design of materials with a tunable response and unprecedented properties. Granular chains, in which nonlinearity arises from the tensionless Hertzian contact, ${ }^{14}$ are perhaps the best example. Applications have been suggested in shock mitigation, ${ }^{15}$ energy localization, ${ }^{15,16}$ stress wave control, ${ }^{17}$ rectification, ${ }^{18}$ and in the design of nonlinear acoustic lenses ${ }^{19}$ among others.

The goal of the present work is to investigate experimentally, numerically, and theoretically the nonlinear response of a 1D NLs formed by identical repelling magnets (see Fig. 1), in which the nonlinearity comes from the repulsive force between magnets. Despite the vast amount of literature appearing in recent decades on NLs, little attention has been given to the dynamics of this system. A recent work by Bernard et $a l .{ }^{20}$ conducted a numerical study of a chain of magnets and demonstrated the existence of band gaps in the linear spectrum and chaotic behavior in the nonlinear regime. The paper by Russel et al. ${ }^{21}$ studied the propagation of moving breathers in a chain of magnetically coupled pendula.

\footnotetext{
a) Author to whom correspondence should be addressed. Electronic mail: daraio@ethz.ch
}

However, the propagation of solitary waves in a chain of magnetic particles has not been studied to date.

The nonlinear magnetic potential between particles shares some key characteristics with the potentials used to describe atomic forces, e.g., the Toda and Lennard-Jones potentials. Both systems present a blow up of the repulsive force when the separation distance between particles tends to zero. Such lattices have been studied theoretically by Friesecke et $a l^{22,23}$ who rigorously proved that they support the propagation of solitary waves with a profile which depends on the amplitude. In particular, the waves present a $\operatorname{sech}^{2}$ profile in the low energy regime and feature atomic scale localization in the high energy regime, i.e., the solitary wave is localized in a single lattice spacing. This characteristic could find potential applications in the design of devices for energy trapping and localization and in the design of novel acoustic lenses. ${ }^{19}$ Since this magnetic system is relatively easy to build and measure, it could be instrumental as a testbed to obtain experimental evidence of important problems in solid state physics.

The paper is organized as follows. Section II describes the lattice and presents the numerical model used for the study. The experimental setup is presented in Sec. III. In Sec. IV, we derive experimentally the interaction potential governing the lattice dynamics. This potential is examined in Sec. V, where we give the theoretical profiles for the solitary waves in the high and low energy regimes. The experimental

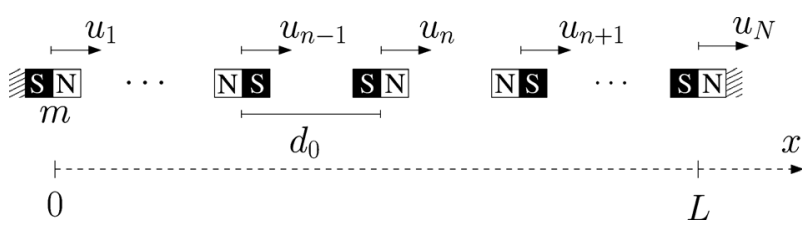

FIG. 1. The chain is composed of $N$ identical magnets with mass $m$. The length of the chain is denoted by $L, d_{0}=L /(N-1)$ denotes the initial spacing between particles, and $u_{n}$ is the displacement from the equilibrium position. 
and numerical results are presented and discussed in Sec. VI and we conclude the article in Sec. VII with a summary of the results and directions for future work.

\section{LATTICE DESCRIPTION AND NUMERICAL MODEL}

We consider a 1D nonlinear lattice composed of $N$ identical magnets with mass $m$, aligned along the $x$-axis such that each magnet repels the adjacent neighbor (see Fig. 1).

Since the interaction force is purely repulsive, it is necessary to fix the position of the magnets on the extremities to obtain a stable state of the system, otherwise the chain would ideally expand to infinity. If the distance between the first and last element of the chain (measured from center to center) is fixed to $L$ then the separation distance at equilibrium between adjacent magnets is $d_{0}=L /(N-1)$. The initial positions of the particle centers are then $x_{n}=(n-1) d_{0}, n=1,2, \ldots, N$. Here, we consider only motion along the $x$-direction and restrict rotation. Thus, the dynamics of the lattice is fully described in terms of the longitudinal displacements $u_{n}(t)$. From $u_{n}$ we also define the relative displacement between adjacent particles $r_{n}(t)$ as

$$
r_{n}(t)=u_{n+1}(t)-u_{n}(t) .
$$

The nonlinear interaction potential between particles is denoted by $V\left(r_{n}\right)$ and the interaction force by $F\left(r_{n}\right)$. These two quantities are related by

$$
F\left(r_{n}\right)=-\frac{\mathrm{d} V\left(r_{n}\right)}{\mathrm{d} r_{n}}=-V^{\prime}\left(r_{n}\right) .
$$

Only nearest neighbor interactions are considered. This assumption is justified by the rapid decay of the repulsive force with the distance (see Sec. IV). Thus, the forces acting on particle $n$ are the repulsive forces exerted by the left and right adjacent particles, respectively, $V^{\prime}\left(u_{n}-u_{n-1}\right)$ and $V^{\prime}\left(u_{n+1}-u_{n}\right)$, and the friction force, that is modeled as viscous damping. From these considerations, the equation of motion of magnet $n$ takes the form of a Fermi-Pasta-Ulam problem $^{1}$

$$
m \ddot{u}_{n}=V^{\prime}\left(u_{n+1}-u_{n}\right)-V^{\prime}\left(u_{n}-u_{n-1}\right)-\alpha \dot{u}_{n} .
$$

Here, $\ddot{u}_{n}, \dot{u}_{n}$, and $\alpha$ denote acceleration, velocity, and damping coefficient, respectively. The form of the magnetic potential $V$ is specified later in Sec. IV.

\section{EXPERIMENTAL SETUP}

The lattice is assembled using $N=26$ identical $\mathrm{NdFeB}$ ring magnets, type R-19-09-06-N from Supermagnete. Magnets are axially magnetized with magnetization grade N42. They have a manufacturer specified mass $m=10.5 \mathrm{~g}$, inner diameter $9.5 \mathrm{~mm}$, outer diameter $19.1 \mathrm{~mm}$, and height $6.4 \mathrm{~mm}$. To maintain the magnetic particle alignment along the $x$-axis and properly oriented (NS-SN), as in Fig. 1, they are placed around a circular rod. Notice that in the absence of the rod the magnets would tend to re-organize forming a chain NS-NS-NS-NS-. The chosen rod diameter was $9.4 \mathrm{~mm}$ : large enough to prevent rotations yet sufficiently small to allow the magnets to move freely along the rod axis. The rod is fixed at the extremities and supported by two thin plates in the middle of the chain to prevent bending due to its own weight. The rod was made of polyoxymethylene (POM), which offers a combination of low friction and a reasonably high stiffness compared to other non-metals.

Magnets 1 and 26 are fixed at distance of $L=1.25 \mathrm{~m}$, which generates an initial spacing between particles $d_{0}=5 \mathrm{~cm}$. To excite the chain, we give an initial displacement $u_{0}$ to magnet 1 , while magnet 2 is held fixed with a movable plate, see Fig. 2. By moving up the plate, magnet 2 starts moving to the right, thus introducing a compression wave in the chain. The possible excitation range is a priori $u_{0} \in\left(0, d_{0}-h\right)$, with $h=6.4 \mathrm{~mm}$ the magnets thickness. However, due to friction losses, it was necessary to excite the system with relatively high amplitude in order for the generated waves to measurably traverse the chain. For this reason, the experimental study is limited to high amplitude excitations. In particular, we have considered three amplitudes $u_{0}=3 \mathrm{~cm}, u_{0}=3.5 \mathrm{~cm}$, and $u_{0}=4 \mathrm{~cm}$.

The motion was measured using a Digital Image Correlation (DIC) System from Correlated Solutions. We use a high speed camera model Phantom from Vision Research to record the motion of particles 13 to 17 with a frame rate of $9000 \mathrm{fps}$. To help the system track the particle motion, it was necessary to glue speckle patterns to the magnets, as shown in Fig. 2. The video files were post-processed with the VIC software to extract the displacement and particle velocities. The DIC technique is not intrusive and it enables us to simultaneously measure a large region of the chain. Furthermore, the large displacements of the particles (of the order of centimeters) make this technique particularly well suited for the study.

\section{INTERACTION POTENTIAL}

It is usual to approximate the repelling force $F_{R}$ between two magnets by modeling the magnets as magnetic

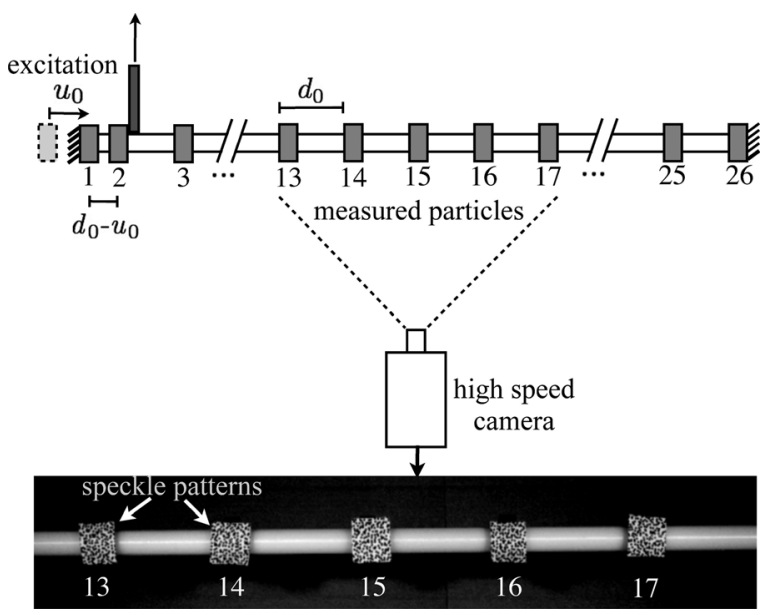

FIG. 2. Schematics of the experimental setup. The chain is formed by 26 ring magnets placed around a plastic rod. To excite the system we give an initial displacement $u_{0}$ to magnet 1 . A high speed camera records the motion of magnets 13 to 17 and DIC is used to extract the displacement and velocity of the particles. The lower picture shows a snapshot of the system at rest. 
dipoles. $^{20,21}$ For identical magnets with magnetic moment $M$ the dipole-dipole repulsive force is given by ${ }^{24}$

$$
F_{R}(d)=\frac{3 \mu_{0} M^{2}}{2 \pi d^{4}}
$$

where $d$ is the separation distance and $\mu_{0}$ is the magnetic constant. However, such assumption is only valid when the separation distance is large compared to the size of the magnets. Since experiments in the present work consider separation distances of the same order as the magnet dimensions, it is more appropriate to estimate $F_{R}$ based on experimental measurements in this range. This force was measured using a simple but effective experiment. Placing the rod vertically, a magnet was fixed to the ground and a second magnet was suspended above the first one. Then 12 different masses where placed on the second magnet, and by measuring the separation distances, we obtained the $F_{R}$ vs $d$ relationship. The measured values are represented by circles in Fig. 3. We assumed that this relationship has a general form similar to Eq. (4)

$$
F_{R}(d)=A d^{p}
$$

with $A>0$ and $p<0$. The experimental data were plotted in logarithmic scale and fitted linearly to calculate $A$ and $m$ [see the inset in Fig. 3(a)], obtaining $A=6.25 \times 10^{-5}$ and $p=-2.73$. As a comparison, we show in Fig. 3(a) the dipole-dipole interaction force [Eq. (4)], with $M=1.29 \mathrm{~J} / \mathrm{T}$ derived from the magnetic properties given by the manufacturer (dashed line). The figure shows that the fitted relationship (solid line) represents very well the behavior observed experimentally, while the dipole-dipole interaction law clearly fails for $d<2.5 \mathrm{~cm}$. Thus, only the experimental repulsive force [Eq. (5)] will be considered in the remainder of this work.

The total interaction force $F$ can be written in terms of the relative displacement $r_{n}$ as

$$
F\left(r_{n}\right)=A\left(d_{0}+r_{n}\right)^{p}-F_{0}
$$

with $d_{0}+r_{n}=d$ and $F_{0}=F_{R}\left(d=d_{0}\right)$ the force necessary to maintain the system at equilibrium. The potential $V\left(r_{n}\right)$ is obtained from Eqs. (2) and (6) as

$$
V\left(r_{n}\right)=-\frac{A\left(d_{0}+r_{n}\right)^{p+1}}{p+1}+F_{0} r_{n} .
$$

$V\left(r_{n}\right)$ is represented in Fig. 3(b). The potential has a minimum at $r_{n}=0$ (equilibrium position) and a vertical asymptote at $r_{n}=-d_{0}$.

\section{THEORETICAL WAVE PROFILES}

As we will demonstrate in the following section (Sec. VI), the magnetic chain exhibits a similar behavior to the generic nonlinear lattices described in Refs. 22 and 23, supporting solitary waves with a profile and wave speed that depend on the amplitude. The key physical requirements cited in Refs. 22 and 23 are (i) that the potential is minimized
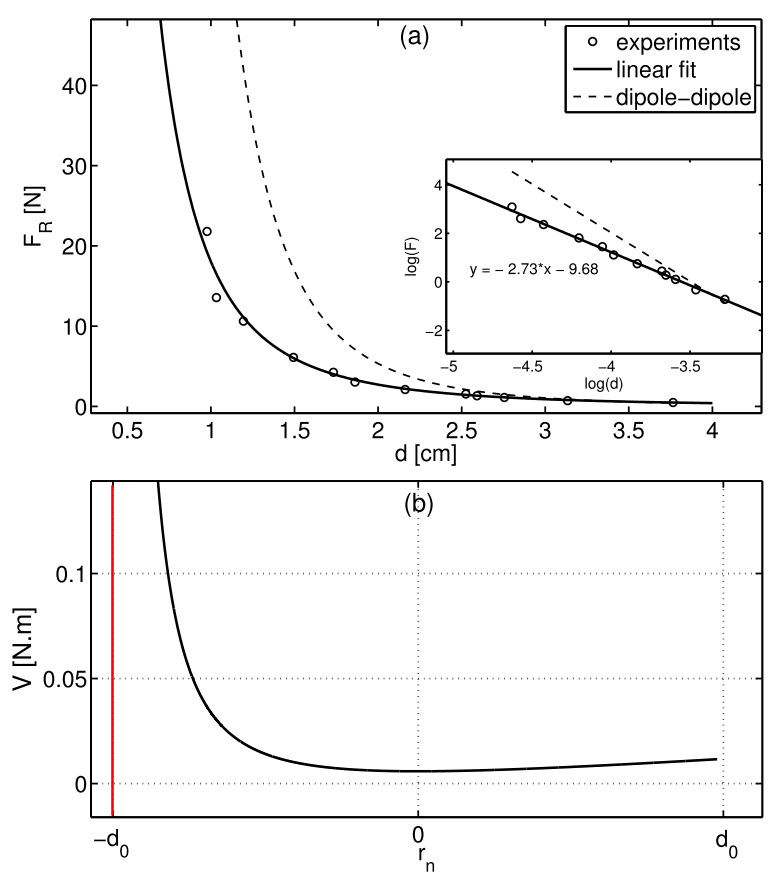

FIG. 3. Magnetic repulsive force law. (a) Measured (circles) and fitted (solid line) repulsive force $F_{R}$ as a function of the distance between magnets $d$. Dashed lines represent the dipole-dipole repulsive force, given by Eq. (4). (b) Potential as a function of the relative displacement $r_{n}$, given by Eq. (7).

when neighboring particles are at some equilibrium distance and (ii) the potential tends to infinity as the distance between particles tends to zero. Mathematically this is satisfied by the following three conditions: ${ }^{23}$

(c1) minimum at zero, $V \geq 0, V^{\prime}(0)=0, V^{\prime \prime}(0)>0$,

(c2) growth, $V\left(r_{n}\right) \geq a\left(\overline{d_{0}}+r_{n}\right)^{-1}$ for some $a>0$ and all $r$ close to $-d_{0}$,

(c3) hardening, $V^{\prime \prime \prime}\left(r_{n}\right)<0$ in $\left(-d_{0}, d_{0}\right)$.

A simple analysis demonstrates that the potential given by Eq. (7) fulfills the above conditions. Other examples of potentials verifying these properties are the Lennard-Jones potential, the Toda potential or, as recently shown, the potential governing the dynamics of tensegrity lattices. ${ }^{25}$

In the low energy regime Friesecke and $\mathrm{Pego}^{22}$ proved that the continuous limit of the relative displacement profile $r(\xi)$ tends monotonically to $r(\xi)=r_{a}(\xi)+\mathcal{O}\left(\varepsilon^{4}\right)$, with

$$
r_{a}(\xi)=\frac{V^{\prime \prime}(0)}{V^{\prime \prime \prime}(0)}\left(\frac{\varepsilon}{2} \operatorname{sech}\left(\frac{\varepsilon \xi}{2}\right)\right)^{2}
$$

In Eq. (8), $\xi$ is the propagation coordinate centered at the maximum of $r_{a}, \varepsilon=\left(24\left(c-c_{s}\right) / c_{s}\right)^{1 / 2}$ is a small parameter, $c$ is the solitary wave speed, and $c_{s}=\sqrt{V^{\prime \prime}(0) / m}$ is the maximum group velocity of the linear waves, also called the sound speed. More precisely $r_{a}(\xi)$ is the solution of a $\mathrm{KdV}$ equation with derivatives of the potential as coefficients. ${ }^{22}$

In the high energy regime Friesecke and Matthies ${ }^{23}$ demonstrated that the profile of the solitary wave is concentrated within a single lattice spacing (only one particle is moving) and has the following form: 


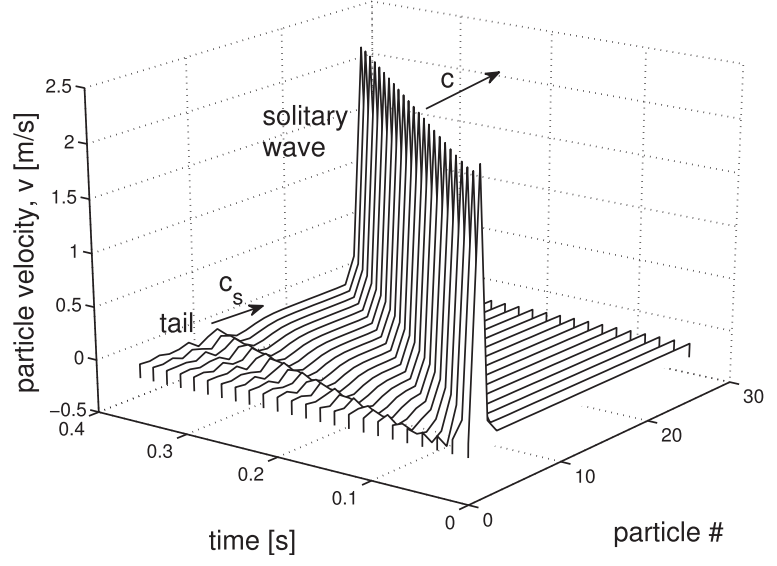

FIG. 4. Particle velocity in the chain at different time steps.

$$
r_{b}(\xi)=\left\{\begin{array}{cc}
|\xi|-d_{0}, & \text { if }|\xi|<d_{0} \\
0, & \text { elsewhere }
\end{array}\right.
$$

Moreover, it was shown that the velocity of the solitary wave tends to the sound speed in the low energy limit

$$
\lim _{r_{n} \rightarrow 0} c=c_{s},
$$

and that it grows unboundedly in the high energy limit

$$
\lim _{r_{n} \rightarrow-d_{0}} c=\infty .
$$

It is worth mentioning that the latter limit is not reachable in practice due to the finite size of the magnets.

\section{EXPERIMENTAL AND NUMERICAL RESULTS}

\section{A. Generation of solitary waves in the lattice}

To have a global picture of the lattice behavior, Fig. 4 represents the velocity of each particle $v_{n}=\dot{u}_{n}$ as a function of time. The geometrical parameters for the simulation are those of the experimental setup. The figure corresponds to the excitation $u_{0}=3.5 \mathrm{~cm}$, but it can be used to describe the lattice behavior for any excitation amplitude, although the exact pulse width and wave speed will vary. The excitation of the lattice generates a solitary wave traveling at speed $c$. The wave is followed by a low amplitude oscillatory tail traveling at speed $c_{s}$. The origin of this tail is the excitation of the linear part of the system. The velocity of the tail was calculated from the time taken to cross the lattice. It was found to be $c_{s}=1.62 \mathrm{~m} / \mathrm{s}$, which is very close to the theoretical value $c_{s}=1.64 \mathrm{~m} / \mathrm{s}$ (see Sec. V). Such small deviation can be attributed to numerical errors and to the finite size of the system. It was also observed that $c_{s}$ was independent from the excitation amplitude, proving its linear nature.

Figure 5 shows the displacements (top panels) and velocities (bottom panels) of particles 13 to 17 for $u_{0}=4 \mathrm{~cm}$ [Figs. 5(a) and 5(d)], $u_{0}=3.5 \mathrm{~cm}$ [Figs. 5(b) and 5(e)], and $u_{0}=3 \mathrm{~cm}$ [Figs. 5(c) and 5(f)]. Solid, dashed, and dotted lines represent experimental results, numerical results with damping, and numerical results without damping, respectively.

The effects of attenuation are evident if one compares experiments and simulations without damping. The amplitudes in experiments are significantly lower, further decreasing with the particle number. To take into account the losses in the system we adjusted the damping coefficient to $\alpha=0.024$. This value was chosen such that the maximum wave velocity of particle 13 coincided with the one observed experimentally. With the addition of dissipation in the simulations, the agreement with the experimental results, for both displacements and particle velocities, is remarkably good for $u_{0}=4 \mathrm{~cm}$, and is also very good for $u_{0}=3.5 \mathrm{~cm}$. As the excitation amplitude decreases, we began to notice differences in the wave profiles between experiments and numerical simulations, as seen in the displacements profiles for $u_{0}=3 \mathrm{~cm}$. The observed discrepancies can be attributed to the choice of
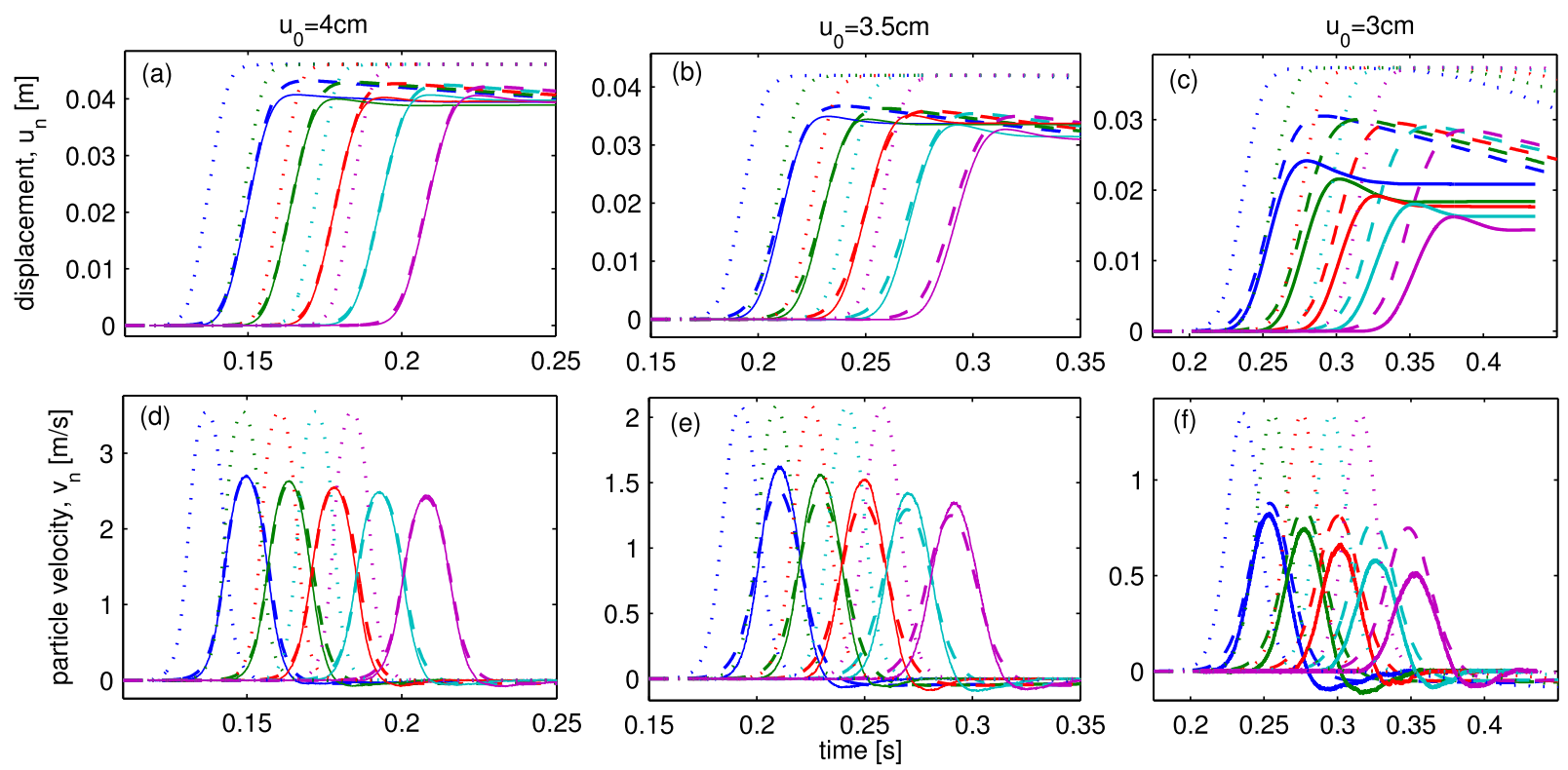

FIG. 5. (a)-(c) are the displacements for $u_{0}=4 \mathrm{~cm}, u_{0}=3.5 \mathrm{~cm}$, and $u_{0}=3 \mathrm{~cm}$, respectively. (d)-(f) are the particle velocities for $u_{0}=4 \mathrm{~cm}, u_{0}=3.5 \mathrm{~cm}$, and $u_{0}=3 \mathrm{~cm}$, respectively. Solid, dashed, and dotted lines represent experimental data, simulations with damping, and simulations without damping, respectively. The experimental time axis was shifted so the arrival time of the wave at sensor 13 coincided in experiments and numerical simulations with dissipation. 


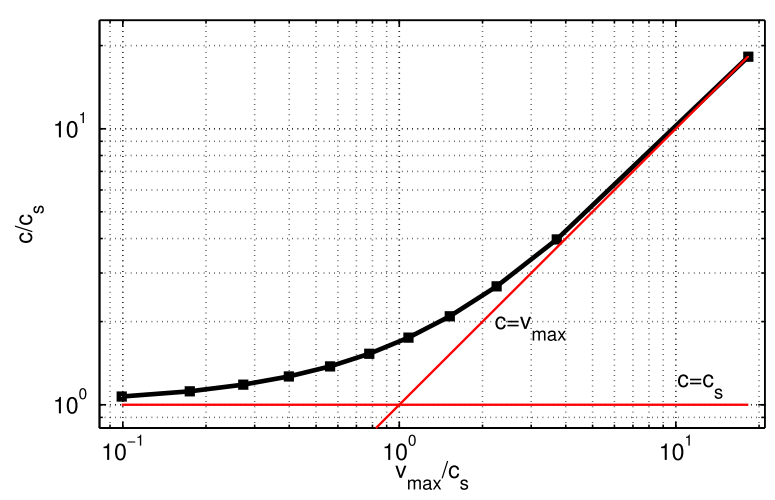

FIG. 6. Velocity of the solitary wave $c$ as a function of the maximum particle velocity $v_{\max }$. $\mathrm{c}$ is bounded by $c_{s}$ at low amplitudes and by $v_{\max }$ at high amplitudes. Squares represent the wave speeds computed from numerical simulations.

damping model. Unlike the model we used here, friction losses are most likely nonlinearly dependent on the particle velocity, with more significant effects on small amplitude waves. ${ }^{26}$ Namely, friction is usually accompanied by stiction, defined as the static friction force that the particles need to overcome to be able to move. This phenomenon is visible in Fig. 5(c) where it is observed that particles stop moving suddenly ( $u_{n}$ remains constant) after the solitary wave has passed.

\section{B. Analysis of the solitary waves}

The wave speed $c$ can be calculated numerically from the time of flight of the solitary wave between two particles. Due to the fact that the solitary wave needs a certain time to completely detach from the tail and to stabilize, see Fig. 4, we consider a longer chain, composed of $N=500$ magnets. $c$ was measured between particles 150 and 450. The lower site, 150, was chosen to ensure that the soliton is totally detached from the tail at the lowest amplitude excitation, that is, when $c \approx c_{s}$. No losses were considered in this case. The results are shown in Fig. 6. The wave speed is plotted versus the maximal particle velocity $v_{\max }$ in the range $v_{\max } \in[0.16,30] \mathrm{m} / \mathrm{s}$. These values correspond to initial displacements $u_{0} \in[0.15,4.85] \mathrm{cm}$. At low amplitudes $c$ tends to $c_{s}$ which is consistent with the theoretical prediction, see Eq. (10). At high amplitudes, the propagation speed grows very fast, also in agreement with the theory, see Eq. (11). We notice that in the high energy limit the velocity of the solitary waves coincide with the maximum particle velocity $\left(c \approx v_{\max }\right)$. This indicates that the energy is being carried by a single particle in the chain, or in other words, that the wave is localized in a single lattice spacing. This will be seen more clearly in the following.

The dependence of the wave profile on the amplitude is now analyzed. Fig. 7 shows the numerical and theoretical relative displacements for excitation amplitudes ranging from $u_{0} / d_{0}=0.2$ [Fig. 7(a)] to $u_{0} / d_{0}=0.98$ [Fig. 7(f)]. In Figs. 7(c)-7(e), we also show the experimental profiles extracted from Figs. 5(a)-5(c), respectively.

At low amplitudes [Figs. 7(a) and 7(b)], the numerical profile is very well described by the theoretical profile $r_{a}$ given by Eq. (8). The numerical and analytical profiles practically coincide in Fig. 7(a). With increasing amplitude [Figs. 7(c) to 7(e)] $r_{a}$ is progressively no longer a good
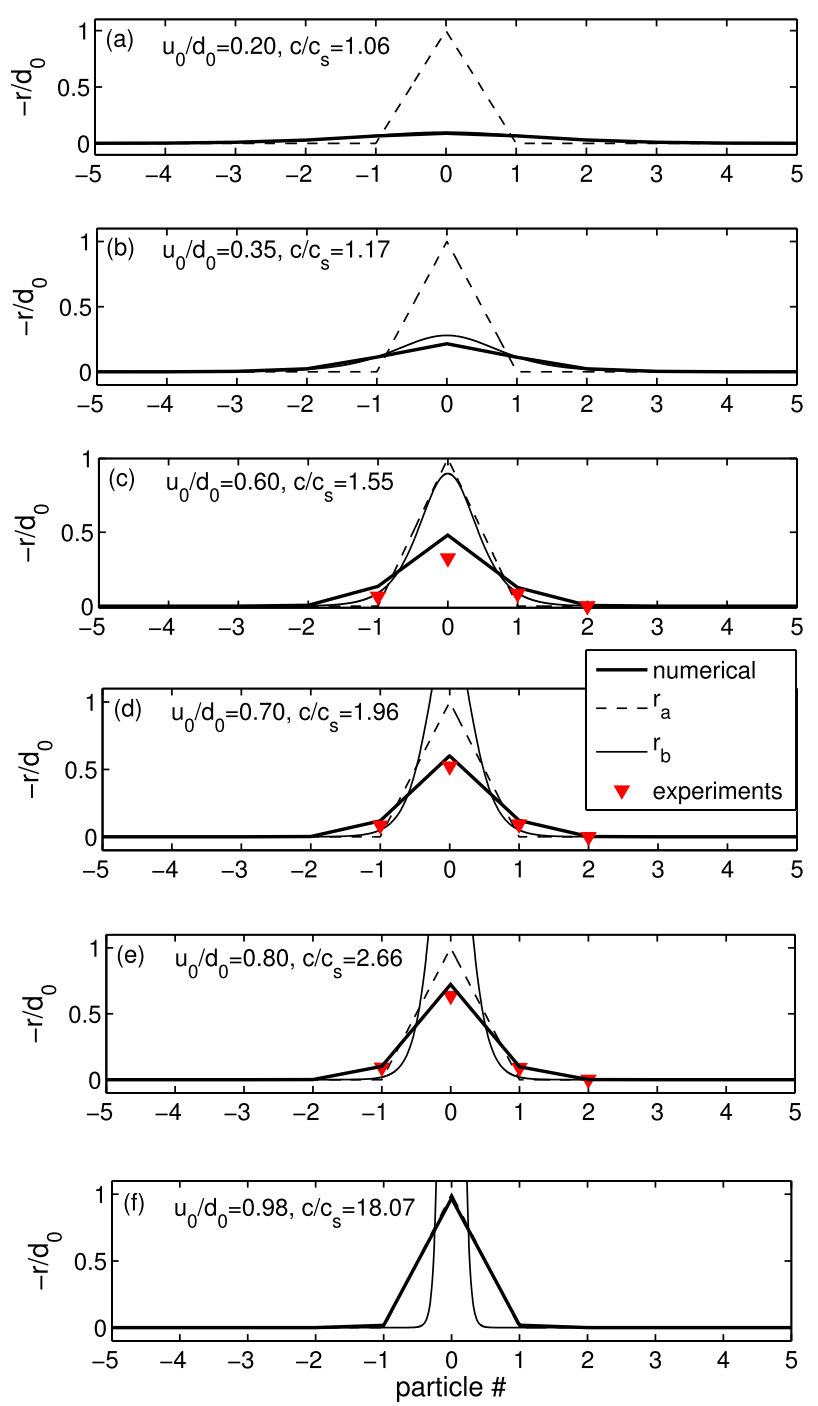

FIG. 7. Relative displacements profiles for different excitations $u_{0}$. (a) $u_{0} / d_{0}=0.2$, (b) $u_{0} / d_{0}=0.35, \quad$ (c) $u_{0} / d_{0}=0.60, \quad$ (d) $u_{0} / d_{0}=0.70$, (e) $u_{0} / d_{0}=0.80$, (f) $u_{0} / d_{0}=0.98$. Thick solid lines represent numerical profiles. Thin solid lines represent the low energy theoretical profile given by Eq. (8). Dashed lines represent the high energy theoretical profile given by Eq. (9). Triangles in (c)-(e) are experimental data.

approximation and the numerical solution tends to the high energy analytical profile $r_{b}$ given by Eq. (9). The experimental results (triangles) are in good agreement with the numerical ones and corroborate the narrowing of the solitary wave with the amplitude. We note nevertheless that due to the dissipation, the experimental profiles have smaller amplitude than the simulated ones. At very high amplitude [Fig. 7(f)], the profile of the solitary wave is almost localized in a single lattice spacing and is practically indistinguishable from the theoretical profile [Eq. (9)].

\section{CONCLUSIONS AND FURTHER DIRECTIONS}

We have investigated experimentally, numerically, and theoretically the propagation of solitary waves in a 1D nonlinear lattice of repelling magnets. The system belongs to the kind of nonlinear lattices described by Friesecke et al. ${ }^{22,23}$ In the low energy regime $\left(c \approx c_{s}\right)$, the profile of the solitary wave is given by a $\mathrm{KdV}$ soliton with a $\operatorname{sech}^{2}$ shape. In the 
high energy regime $\left(c \gg c_{s}\right)$, the wavelength progressively shrinks, with the limiting case $(c \rightarrow \infty)$ being a hat function with width equal to a single lattice period (atomic scale localization). These two cases have been observed in the numerical simulations. The system has been studied experimentally using the DIC technique but, due to friction losses, the experimental study has been limited to the high energy regime. Experiments are in very good agreement with numerical simulations and have permitted observation of the shrinking width of the solitary wave with increasing amplitude. In future studies, we plan to build alternative systems in order to reduce friction losses and also to extend the study to two-dimensions. In addition to their use as a toy model for the study of fundamental nonlinear dynamical systems, such systems could find potential applications in energy mitigation and localization or in the design of acoustic lenses capable of omitting very narrow pulses.

\section{ACKNOWLEDGMENTS}

This work was supported by the Army Research Office MURI, Project No. W911NF0910436.

${ }^{1}$ E. Fermi, J. Pasta, and S. Ulam, Los Alamos Scientific Laboratories, Los Alamos, Report No. LA1940, 1955.

${ }^{2}$ N. J. Zabusky and M. D. Kruskal, Phys. Rev. Lett. 15, 240 (1965).

${ }^{3}$ P. G. Kevrekidis, IMA J. Appl. Math. 76, 389 (2011).

${ }^{4}$ Y. V. Kartashov, B. A. Malomed, and L. Torner, Rev. Mod. Phys. 83, 247 (2011).
${ }^{5}$ M. Toda, Theory of Nonlinear Lattices, 2nd ed. (Springer, Berlin, 1989). ${ }^{6}$ A. S. Davydov, Solitons in Molecular Systems (Reidel, Kiev, 1985).

${ }^{7}$ M. A. Collins, Chem. Phys. Lett. 77, 342 (1981).

${ }^{8}$ J. Cabaret, V. Tournat, and P. Béquin, Phys. Rev. E 86, 041305 (2012).

${ }^{9}$ V. J. Sánchez-Morcillo, I. Pérez-Arjona, V. Romero-García, V. Tournat, and V. E. Gusev, Phys. Rev. E 88, 043203 (2013).

${ }^{10}$ C. Hoogeboom, Y. Man, N. Boechler, G. Theocharis, P. Kevrekidis, I. Kevrekidis, and C. Daraio, Europhys. Lett. 101, 44003 (2013).

${ }^{11}$ N. Boechler, G. Theocharis, S. Job, M. Porter, P. Kevrekidis, and C. Daraio, Phys. Rev. Lett. 104, 244302 (2010).

${ }^{12}$ G. Theocharis, N. Boechler, P. G. Kevrekidis, S. Job, M. A. Porter, and C. Daraio, Phys. Rev. E 82, 056604 (2010).

${ }^{13}$ C. Chong, P. Kevrekidis, G. Theocharis, and C. Daraio, Phys. Rev. E 87, 042202 (2013).

${ }^{14}$ V. F. Nesterenko, Dynamics of Heterogeneous Materials (Springer, Berlin, 2001).

${ }^{15}$ C. Daraio, V. F. Nesterenko, E. B. Herbold, and S. Jin, Phys. Rev. Lett. 96, 058002 (2006).

${ }^{16}$ D. K. Campbell, S. Flach, and Y. S. Kivshar, Phys. Today 57(1), 43 (2004).

${ }^{17}$ A. Leonard and C. Daraio, Phys. Rev. Lett. 108, 214301 (2012).

${ }^{18}$ N. Boechler, G. Theocharis, and C. Daraio, Nature Mater. 10, 665 (2011).

${ }^{19}$ A. Spadoni and C. Daraio, Proc. Natl. Acad. Sci. U. S. A. 107, 7230 (2010).

${ }^{20}$ B. Bernard, B. Owens, and B. Mann, J. Vib. Acoust. 135, 041005 (2013).

${ }^{21}$ F. M. Russell, Y. Zolotaryuk, J. C. Eilbeck, and T. Dauxois, Phys. Rev. B 55, 6304 (1997).

${ }^{22}$ G. Friesecke and R. Pego, Nonlinearity 12, 1601 (1999).

${ }^{23}$ G. Friesecke and K. Matthies, Physica D 171, 211 (2002).

${ }^{24}$ K. W. Yung, P. B. Landecker, and D. D. Villani, Magn. Electr. Sep. 9, 39 (1998).

${ }^{25}$ F. Fraternali, L. Senatore, and C. Daraio, J. Mech. Phys. Solids 60, 1137 (2012).

${ }^{26}$ H. Olsson, K. Astrom, C. C. de Wit, M. Gafvert, and P. Lischinsky, Eur. J. Control 4, 176 (1998) 